\title{
On polynomials of spanning trees *
}

\author{
Fan Chung $^{\dagger}$ \\ University of California, San Diego \\ La Jolla, CA 92093-0112
}

\author{
Chao Yang \\ University of Pennsylvania \\ Philadelphia, PA 19104
}

August 29, 2000

\begin{abstract}
The Kirchhoff polynomial of a graph $G$ is the sum of weights of all spanning trees where the weight of a tree is the product of all its edge weights, considered as formal variables. Kontsevich conjectured that when edge weights are assigned values in a finite field $\mathbb{F}_{q}$, for a prime power $q$, the number of zeros of the Kirchhoff polynomial of a graph $G$ is just a polynomial function of $q$. Stanley verified this conjecture for some families of graphs and further proposed several conjectures. In this paper, we derive and prove explicit formulas for certain graphs, thus confirming Stanley's conjectures. We also consider several extensions and generalizations of the conjecture of Kontsevich.
\end{abstract}

\section{Introduction}

Let $G=(V(G), E(G))$ be a graph $G$ with vertex set $V(G)$ and edge set $E(G)$. We associate with each of its edge $e$ a variable $X_{e}$. The Kirchhoff polynomial of $G$, denoted by $\operatorname{Kir}(G)$, is defined to be

$$
\operatorname{Kir}(G)=\sum_{T} \prod_{e} X_{e}
$$

where the sum is taken over all spanning trees $T$ of $G$, and the product is taken over all the edges of $T$. A zero of $\operatorname{Kir}(G)$ over a ring $\mathrm{R}$ is an assignment of values in $\mathrm{R}$ to $X_{e}$ for all $e$ in $E(G)$ such that the Kirchhoff polynomial has value 0 . Kontsevich [5] conjectured that the number of zeros for a Kirchhoff

\footnotetext{
${ }^{*}$ To appear in Annals of Combinatorics.

${ }^{\dagger}$ Research supported in part by NSF Grant No. DMS 98-01446
} 
polynomial of a given graph $G$ over a finite field $\mathbb{F}_{q}$, for a prime power $q$, is a 'universal' polynomial function in $q$.

This problem can be restated as follows:

Suppose that the variables $\left\{X_{e}\right\}_{e \in E(G)}$ are independently and uniformly distributed over the finite field $\mathbb{F}_{q}$. The problem of interest is to compute the probability function $\operatorname{Pk}(G)=\operatorname{Prob}(\operatorname{Kir}(G)=0)$ over a field with $q$ elements, and to determine whether $\operatorname{Pk}(G)$ is a polynomial in $q^{-1}$. If $\operatorname{Pk}(G)$ is a polynomial in $\mathbb{Z}\left[q^{-1}\right]$, we call it the probabilistic Kirchhoff polynomial of $G$, and we say that the graph $G$ is admissible over $\mathbb{F}_{q}$.

For example, for $G=K_{3}$, the complete graph on three vertices, we use $X_{1}, X_{2}$ and $X_{3}$ to denote the variables associated with its three edges. Its Kirchhoff polynomial and its probabilistic Kirchhoff polynomial over $\mathbb{F}_{p^{k}}$ are

$$
\begin{aligned}
& \operatorname{Kir}\left(K_{3}\right)=X_{1} X_{2}+X_{2} X_{3}+X_{3} X_{1} \\
& \operatorname{Pk}\left(K_{3}\right)=\operatorname{Prob}\left(\operatorname{Kir}\left(K_{3}\right)=0\right)=\frac{1}{q}
\end{aligned}
$$

The problem of determining the probabilistic Kirchhoff polynomial for complete graphs has been previously studied in the literature. By using the matrix-tree theorem, it is not difficult to see that the probabilistic Kirchhoff polynomial for a complete graph $K_{n}$ over $\mathbb{F}_{q}$ is exactly the probability of an $(n-1) \times(n-1)$ matrix with entries in a finite field being singular. The probabilistic Kirchhoff polynomial for $K_{n}$ over a finite field $\mathbb{F}_{q}$ is

$$
\operatorname{Pk}\left(K_{n}\right)=1-\prod_{j=1}^{\left\lfloor\frac{n}{2}\right\rfloor}\left(1-q^{-2 j+1}\right) .
$$

The evaluation of $\operatorname{Pk}\left(K_{n}\right)$ over a finite field $\mathbb{F}_{q}$, for odd prime power $q$, can be traced to Carlitz [3] in 1954. A simple proof was later found by MacWilliams [6] in 1969. Brent and McKay [2, Thm. 5.1, 1988] investigated $\operatorname{Pk}\left(K_{n}\right)$ over the ring $\mathbb{Z}_{m}$. They also evaluated ranks of random matrices over $\mathbb{Z}_{m}$ (see[1]). Recently, Stanley [7] gave another proof for (2) using orthogonal geometries over $\mathbb{F}_{q}$. He also solved the problems for $K_{n}-K_{1, s}$ (deleting $K_{1, s}$ from $K_{n}$ ) and conjectured formulas for the probabilistic Kirchhoff polynomials of $K_{n}-K_{3}, K_{n}-K_{4}$, and $K_{n}-K_{5}$. We will verify Stanley's conjecture by determining $\operatorname{Pk}\left(K_{n}-K_{m}\right)$ for general $m$. We will also consider several extensions of Kontsevich's conjecture concerning, for example, the probability of the Kirchhoff polynomial assuming a nonzero value as well as another direction on the probability distribution of the rank function of the associated matrices. Further remarks, examples, problems and conjectures are discussed in the last section. 
Stembridge [8] gave a reduction algorithm for computing the probabilistic Kirchhoff polynomials. His MAPLE program verified Kontsevich's conjecture for all graphs with no more than 12 edges. He also proved a number of results on minimal counterexamples for the Kontsevich's conjecture. For example, he showed that such a minimal graph cannot be 2-connected. This implies that outerplanar graphs have probabilistic Kirchhoff polynomial (which was proved independently by one of the authors [10]).

\section{Preliminaries}

For simplicity, we here consider a simple loopless graph $G$ on $n$ vertices (although the definitions and the theorems can be easily extended to the general case). The combinatorial Laplacian $L(G)$ of $G$ is the $n \times n$ symmetric matrix defined by

$$
L_{i j}(G)= \begin{cases}-x_{e}, & \text { if } i \neq j \text { and } e \text { has vertices } v_{i} \text { and } v_{j} \\ 0, & \text { if } i \neq j \text { and there is no edge with vertices } v_{i} \text { and } v_{j} \\ \sum_{e} x_{e}, & \text { if } i=j \text { and } e \text { ranges over all edges incident to } v_{i} .\end{cases}
$$

The $i$-th reduced Laplacian $\tilde{L}_{i}(G)$ is the matrix $L(G)$ with its $i$-th row and $i$-th column removed. The classical matrix-tree theorem states (see $[4,9])$ :

$$
\operatorname{Kir}(G)=\operatorname{det}\left(\tilde{L}_{i}(G)\right)
$$

for any $i$ (independent of the choice of $i$ ). Thus, any $n \times n$ symmetric matrix $M$ can be associated with a reduced Laplacian of some graph $G$ as follows: The graph $G$ has the combinatorial Laplacian $\left[\begin{array}{ll}M & u \\ u^{*} & z\end{array}\right]$, where $u=-M \cdot \mathbf{1}$, $u^{*}$ is the transpose of $u, z=-u^{*} \cdot \mathbf{1}$, and $\mathbf{1}$ is the column vector $(1,1, \ldots, 1)^{*}$.

We say a graph $G$ is strongly admissible over a field $\mathcal{F}$ if for any integer $r$, the probability

$$
p(r, G)=\operatorname{Prob}(\operatorname{rank}(\tilde{L}(G))=r)
$$

is a universal polynomial function in $1 /|\mathcal{F}|$.

Remark 1: For a graph on $n$ vertices, we have

$$
\operatorname{Pk}(G)=1-p(n-1, G) .
$$


Remark 2: For a strongly admissible graph $G$, it is natural to consider the following generating function:

$$
\begin{aligned}
\mathcal{H}(G)(X) & =\sum_{r} p(r, G) X^{r} \\
& =\sum_{r} \operatorname{Prob}(\operatorname{rank}(\tilde{L}(G))=r) \cdot X^{r}
\end{aligned}
$$

A graph $G$ is strongly admissible over a field $\mathcal{F}$ with $q=|\mathcal{F}|$ if

$$
\mathcal{H}(G)(X) \in \mathbb{Z}\left[q^{-1}\right][X]
$$

We will use the stronger inductive assumption of strong admissibility to derive the probabilistic Kirchhoff polynomials in the next section.

\section{$3 \quad$ Strongly admissible graphs}

An apex of a graph is a vertex which is adjacent to every other vertex. Stanley [7] gave a recurrence equation for graphs with two or more apexes. For the family $K_{n}-K_{m}$, he conjectured formulas for $\operatorname{Pk}\left(K_{n}-K_{m}\right)$ for $m=3,4,5$. Here we will derive an explicit expression for $\operatorname{Pk}\left(K_{n}-K_{m}\right)$ for general $m$, by proving the following stronger results.

Theorem 3.1 If $G$ has an apex and is strongly admissible, then the graph formed by adding apexes to $G$ is also strongly admissible.

Theorem 3.2 Suppose $G^{\prime}$ is strongly admissible over a field $\mathcal{F}$, with $m$ vertices and at least one apex. Let $M$ denote a reduced Laplacian of $G^{\prime}$ and $q$ denote $|\mathcal{F}|$. Then the graph $G$ on $n$ vertices formed by adding $n-m$ apexes to $G^{\prime}$ satisfies

$$
\begin{aligned}
\operatorname{Pk}(G) & = \\
1 & -\sum_{r=0}^{m} \operatorname{Prob}(\operatorname{rank}(M)=r) \prod_{i=1}^{m-r}\left(1-q^{-n+m+i}\right) \prod_{j=1}^{\left\lfloor\frac{n-2 m+r}{2}\right\rfloor}\left(1-q^{-2 j+1}\right)
\end{aligned}
$$

In particular, we have the following result for graphs $K_{n}-K_{m}$.

Theorem 3.3 For $2 \leq m<n$, the graph $K_{n}-K_{m}$ is strongly admissible over a field $\mathcal{F}$ with the probabilistic Kirchhoff polynomial satisfying: 


$$
\begin{aligned}
& \operatorname{Pk}\left(K_{n}-K_{m}\right)=1- \\
& \sum_{r}\left(\begin{array}{c}
m \\
r
\end{array}\right) q^{-m+r}\left(1-q^{-1}\right)^{r} \cdot \prod_{i=1}^{m-r}\left(1-q^{-n+m+i}\right) \cdot \prod_{j=1}^{\left\lfloor\frac{n-2 m+r}{2}\right\rfloor}\left(1-q^{-2 j+1}\right)
\end{aligned}
$$

where $q=|\mathcal{F}|$.

The proofs for the above theorems rely on a sequence of facts.

Lemma 3.1 For a symmetric matrix $A$ with $\operatorname{det}(A) \neq 0$, the following two matrices are equivalent (i.e., differed by conjugation):

$$
M=\left[\begin{array}{cc}
A & C \\
C^{*} & B
\end{array}\right] \sim\left[\begin{array}{cc}
A & 0 \\
0 & \tilde{B}
\end{array}\right]=\tilde{M}
$$

where $\tilde{B}=B-C^{*} A^{-1} C$.

Proof: For $P=\left[\begin{array}{cc}I & 0 \\ -C^{*} A^{-1} & I\end{array}\right]$, we have $P M P^{*}=\tilde{M}$.

We consider the matrix

$$
M(s, t)=\left[\begin{array}{cc}
C_{s} & B_{s \times t} \\
B^{*} & 0_{t}
\end{array}\right]
$$

where $C_{s}$ is an $s \times s$ random symmetric matrix, (with $s(s+1) / 2$ random variables as entries), $B$ is an $s \times t$ random matrix with $s t$ independent random entries whose transpose is $B^{*}$, and $0_{t}$ is the $t \times t$ zero matrix. We need to examine the probability $p(s, t ; r)$ of the matrix $M(s, t)$ having rank $r$, when all the $s t+s(s+1) / 2$ random variables are independently and uniformly distributed over the finite field $\mathbb{F}_{q}$.

We consider the generating function:

$$
f(s, t)=f(s, t)(X)=\sum_{r=0}^{s+t} p(s, t ; r) X^{r}
$$

which satisfies the following recurrence relation:

\section{Lemma 3.2}

$$
\begin{aligned}
f(0, t) & =1 \\
f(s, t+1) & =q^{-s} f(s, t)+\left(1-q^{-s}\right) f(s-1, t) X^{2}
\end{aligned}
$$


Proof: For $M(s, t+1)$, we consider the last row and its $s$ random variables. With probability $q^{-s}$, all of them are zero and we have $f(s, t)$. Otherwise, let $w$ be a non-zero entry in the block $B$ of $M(s, t)$. We can rearrange the matrix as follows:

$$
\left[\begin{array}{cccc}
\bullet & \bar{\bullet} & \bar{\bullet} & w \\
\bar{\bullet} & \tilde{C}_{s-1} & \tilde{B}_{(s-1) \times t} & \bar{\bullet} \\
\bar{\bullet} & \tilde{B}_{t \times(s-1)}^{*} & t \times t & \overline{0} \\
w & \bar{\bullet} & \overline{0} & 0
\end{array}\right]
$$

where $\bullet$ denotes a random variable with uniform distribution, $\boldsymbol{\bullet}$ denotes a row/column of appropriate size, $\overline{0}$ is a row/column of zeros of some appropriate size, and $\tilde{C}$ denotes an altered $C$.

We consider the submatrix $\left[\begin{array}{cc}\bullet & w \\ w & 0\end{array}\right]$ formed by the entries in four corners of the above matrix. Clearly, it is invertible. We can pivot on this principal submatrix and apply Lemma 3.1. After removing the first and last rows and columns from the above matrix, the resulting matrix is just $M(s-1, t)$. Here we repeatedly use a simple observation that a random variable plus some other value is still random. Hence we have the recurrences in Lemma 3.2.

As an immediate result, for a complete graph $K_{s+1}$, we have

$$
\begin{aligned}
\mathcal{H}\left(K_{s+1}\right) & =f(s, 0) \\
& =q^{-1} f(s-1,1)+\left(1-q^{-1}\right) f(s-1,0) X \\
& =\left[q^{-s}+\left(1-q^{-1}\right) X\right] f(s-1,0)+\left(q^{-1}-q^{-s}\right) X^{2} f(s-2,0)
\end{aligned}
$$

MacWilliams [6] has computed the rank generating function $\mathcal{H}\left(K_{n}\right)$ explicitly. The probability $p\left(r, K_{n}\right)$ that the (reduced) combinatorial Laplacian $L\left(K_{n}\right)$ is of rank $r$ can be expressed as follows:

$$
\begin{aligned}
p\left(2 s, K_{n}\right) & =q^{-n(n-1) / 2} \prod_{i=1}^{s} \frac{q^{2 i}}{q^{2 i}-1} \cdot \prod_{i=0}^{2 s-1}\left(q^{n-i-1}-1\right), \\
p\left(2 s+1, K_{n}\right) & =q^{-n(n-1) / 2} \prod_{i=1}^{s} \frac{q^{2 i}}{q^{2 i}-1} \cdot \prod_{i=0}^{2 s}\left(q^{n-i-1}-1\right),
\end{aligned}
$$

for all $2 s, 2 s+1 \leq n-1$.

From Lemma 3.2 we see that by induction each $f(s, t)(X)$ is a polynomial in $X$ with coefficients in $\mathbb{Z}\left[q^{-1}\right]$. The coefficient of $X^{s+t}$, which is the probability $p(s, t ; s+t)$ that the matrix $M(s, t)$ has full rank, can be expressed by the following: 


\section{Lemma 3.3}

$$
p(s, t ; s+t)= \begin{cases}1-\operatorname{Pk}\left(K_{s+1}\right) & \text { if } t=0 \\ 0 & \text { if } s<t \\ \left(1-\operatorname{Pk}\left(K_{s-t+1}\right)\right) \prod_{i=1}^{t}\left(1-q^{-s+i-1}\right) & \text { otherwise. }\end{cases}
$$

Proof: For $s<t$, we see that $\operatorname{det}(M(s, t))$ is always zero. Thus $M(s, t)$ is never invertible.

For $s \geq t$, if $M(s, t)=\left[\begin{array}{cc}C_{s} & B_{s \times t} \\ B^{*} & 0_{t}\end{array}\right]$ is invertible, the random matrix $B_{s \times t}$ has to be of rank $t$ (with probability $\prod_{i=1}^{t}\left(1-q^{-s+i-1}\right)$ ). We can rearrange $B$ by placing the invertible $t \times t$ sub-block $B_{1}$ as follows:

$$
M(s, t) \sim\left[\begin{array}{ccc}
C_{1} & C_{2} & B_{2} \\
C_{2}^{*} & C_{3} & B_{1} \\
B_{2}^{*} & B_{1}^{*} & 0_{t}
\end{array}\right]
$$

Now we apply Lemma 3.1 by pivoting on the $2 t \times 2 t$ invertible matrix $\left[\begin{array}{cc}C_{3} & B_{1} \\ B_{1}^{*} & 0\end{array}\right]$. Therefore we have

$$
\left[\begin{array}{ccc}
C_{1} & C_{2} & B_{2} \\
C_{2}^{*} & C_{3} & B_{1} \\
B_{2}^{*} & B_{1}^{*} & 0_{t}
\end{array}\right] \sim\left[\begin{array}{ccc}
\tilde{C}_{1} & 0 & 0 \\
0 & C_{3} & B_{1} \\
0 & B_{1}^{*} & 0_{t}
\end{array}\right]
$$

where $\tilde{C}_{1}$ is a random $(s-t) \times(s-t)$ symmetric matrix with $(s-t)(s-t-1) / 2$ independent random variables. Therefore $\tilde{C}_{1}$ is invertible with probability $1-\operatorname{Pk}\left(K_{s-t+1}\right)$.

Proof of Theorem 3.1 : We consider the reduced combinatorial Laplacian $M$ of $G^{\prime}$ by deleting the row and column indexed by an apex. Now, we form the graph $G$ by joining $n-m+1$ apexes to $G^{\prime}$. The reduced combinatorial Laplacian of $G$ can be written as:

$$
\tilde{L}(G)=\left[\begin{array}{cc}
C_{n-m-1} & B_{(n-m-1) \times m} \\
B^{*} & M
\end{array}\right]
$$

where $C$ is random symmetric of size $(n-m-1) \times(n-m-1), B$ is random of size $(n-m-1) \times m$ and $B^{*}$ denotes the transpose of $B$.

If $M$ has rank $r$, the reduced combinatorial Laplacian $\tilde{L}(G)$ can be modified. By pivoting on the $r$ by $r$ invertible submatrix and applying Lemma 3.1, 
we have

$$
\left[\begin{array}{cc}
C_{n-m-1} & B_{(n-m-1) \times m} \\
B^{*} & M
\end{array}\right] \sim\left[\begin{array}{ccc}
\tilde{C}_{n-m-1} & \tilde{B}_{(n-m-1) \times(m-r)} & 0 \\
\tilde{B}^{*} & 0_{m-r} & 0 \\
0 & 0 & M_{r}
\end{array}\right]
$$

where $\tilde{C}$ is a random symmetric matrix, $\tilde{B}$ is a random matrix of size $(n-$ $m-1) \times(m-r)$, and $M_{r}$ denote an $r \times r$ symmetric matrix of full rank. We note that $\tilde{C}$ and $\tilde{B}$ are independent since by adding some value to a random variable it is still independently and uniformly distributed.

Therefore by definition, the rank generating function of $G$ is

$$
\mathcal{H}(G)(X)=\sum_{r} \operatorname{Prob}(\operatorname{rank} M=r) f(n-m-1, m-r) X^{r}
$$

Clearly all the coefficient of $\mathcal{H}(G)$ are polynomials in $\mathbb{Z}\left[q^{-1}\right]$. This proves that $G$ is strongly admissible.

Proof of Theorem 3.2 : We consider the coefficient of $X^{n-1}$ in (10). Using Lemma 3.2, we have

$\operatorname{Pk}(G)=1-\sum_{r=0}^{m} \operatorname{Prob}(\operatorname{rank}(M)=r) \prod_{i=1}^{m-r}\left(1-q^{-n+m+i}\right) \prod_{j=1}^{\left\lfloor\frac{n-2 m+r}{2}\right\rfloor}\left(1-q^{-2 j+1}\right)$

This completes the proof of Theorem 3.2.

As an immediate consequence of Theorem 3.2, we have proved that a complete graph is strongly admissible.

Proof of Theorem 3.3 : The reduced combinatorial Laplacian $M$ of $G^{\prime}=$ $K_{1, n}$, a star on $n+1$ vertices, is just a random diagonal matrix

$$
M=\left[\begin{array}{llll}
a_{1} & & & \\
& a_{2} & & \\
& & \ddots & \\
& & & a_{m}
\end{array}\right]
$$

The rank generating function of $M$ is the following:

$$
\left(q^{-1}+\left(1-q^{-1}\right) Y\right)^{m}=\sum_{r}\left(\begin{array}{c}
m \\
r
\end{array}\right) q^{-m+r}\left(1-q^{-1}\right)^{r} Y^{r} \quad \in \mathbb{Z}[q][Y]
$$


Therefore by Theorem 3.1, the rank generating function of $K_{n}-K_{m}$ is

$$
\begin{aligned}
\mathcal{H}\left(K_{n}-K_{m}\right)(X) & =\sum_{r} \operatorname{Prob}(\text { rank } M=r) f(n-m-1, m-r) X^{r} \\
& =\sum_{r}\left(\begin{array}{c}
m \\
r
\end{array}\right) q^{-m+r}\left(1-q^{-1}\right)^{r} f(n-m-1, m-r) X^{r}
\end{aligned}
$$

where $f$ is defined in (5), and its expression can be found in Lemma 3.2. Therefore, $K_{n}-K_{m}$ is strongly admissible. In particular, we consider the coefficient of $X^{n-1}$ in $\mathcal{H}\left(K_{n}-K_{m}\right)$ and we have

$$
\begin{aligned}
& \operatorname{Pk}\left(K_{n}-K_{m}\right) \\
= & 1-\sum_{r}\left(\begin{array}{c}
m \\
r
\end{array}\right) q^{-m+r}\left(1-q^{-1}\right)^{r} \cdot \prod_{i=1}^{m-r}\left(1-q^{-n+m+i}\right) \cdot\left(1-\operatorname{Pk}\left(K_{n-2 m+r}\right)\right) \\
= & 1-\sum_{r}\left(\begin{array}{c}
m \\
r
\end{array}\right) q^{-m+r}\left(1-q^{-1}\right)^{r} \cdot \prod_{i=1}^{m-r}\left(1-q^{-n+m+i}\right) \cdot \prod_{j=1}^{\left\lfloor\frac{n-2 m+r}{2}\right\rfloor}\left(1-q^{-2 j+1}\right)
\end{aligned}
$$

We remark that for the case of $m=3,4,5$. we have exactly the same formulas for $K_{n}-K_{m}$ as conjectured by Stanley [7].

In the remainder of this section, we consider the graphs $K_{n}-K_{m_{1}, m_{2}, \ldots, m_{s}}$.

Theorem 3.4 For integers $m_{1}, \ldots, m_{s}$ with $\sum_{i} m_{i}<n$, the graph $K_{n}-$ $K_{m_{1}, \ldots, m_{s}}$ is strongly admissible over a finite field $\mathcal{F}$.

\section{Proof:}

First we consider the case that $n^{\prime}=1+\sum_{i} m_{i}$. The reduced combinatorial Laplacian of $G^{\prime}=K_{n^{\prime}}-K_{m_{1}, m_{2}, \ldots, m_{s}}$ can be described as follows:

$$
\left[\begin{array}{cccc}
A_{m_{1}} & & & 0 \\
& A_{m_{2}} & & \\
& & \ddots & \\
0 & & & A_{m_{s}}
\end{array}\right]
$$

where the $A$ 's are random symmetric matrices.

Since the $A$ 's can be viewed as the reduced combinatorial Laplacian of complete graphs, the rank generating function of $G^{\prime}$ satisfies

$$
\mathcal{H}\left(G^{\prime}\right)=\prod_{i=1}^{s} \mathcal{H}\left(K_{m_{i}+1}\right) .
$$


Since the complete graphs are strongly admissible, $G^{\prime}$ is strongly admissible. By Theorem 3.1, the graph $G$, which is formed by adding $\left(n-1-\sum m_{i}\right)$ apexes to $G^{\prime}$, is strongly admissible.

Using MacWilliams' results on $p\left(r, K_{n}\right.$ ) (as shown in (6)) for complete graphs $K_{n}$ 's, we can apply Theorem 3.2 to compute the probabilistic Kirchhoff polynomial of the graph $K_{n}-K_{m_{1}, m_{2}, \ldots, m_{s}}$ explicitly.

\section{The distribution of $\operatorname{Kir}(G)$}

Brent and McKay [2] proposed studying the probability of $\operatorname{Kir}\left(K_{n}\right)=d$ for arbitrary $d$. They observed that the distribution for $\operatorname{Kir}\left(K_{n}\right)=d$ is not uniform over the ring $\mathbb{Z}_{p}$. In fact, for $d$ in $\mathbb{Z}_{p}$, Prob(Kir $\left.\left(K_{n}\right)=d\right)$ depends on $d$ being a quadratic residue or not. Here is the statement in [2] after translating into our notation:

Another open problem is to determine the probability that (for the reduced combinatorial Laplacian of $K_{n}$ which can be viewed as a $(n-1) \times(n-1)$ random matrix $A$ over $\left.\mathbb{Z}_{m}\right) \operatorname{det}(A)$ mod $m$ takes a given value $d$ (where $A$ is the reduced combinatorial Laplacian of $K_{n}$ which can be viewed as a $(n-1) \times(n-1)$ random matrix over $\left.\mathbb{Z}_{m}\right)$. Small examples show that this probability depends on $d$. For example, if $m$ is a prime $p, r=\lceil(n-1) / 2\rceil$, $\left(\frac{d}{p}\right)$ is the Legendre symbol, and

$$
s= \begin{cases}0 & \text { if } \mathrm{p}=2 \text { or } \mathrm{n} \text { is even, } \\ \left(\frac{d}{p}\right)(-1)^{r(p-1) / 2} & \text { otherwise. }\end{cases}
$$

then the probability is

$$
\frac{1}{(p-1)} \cdot \frac{\Pi_{2 r}(1 / p)}{\Pi_{r}\left(1 / p^{2}\right)}\left(1+s p^{-r}\right)
$$

where $\Pi_{k}(x)=\prod_{i=1}^{k}\left(1-x^{i}\right)$.

No proof for (14) was given in [2]. Here we will give a proof for the general case over a finite field:

Theorem 4.1 The Kirchhoff polynomial for $K_{n}$ over a field $\mathbb{F}_{q}$ satisfies

$$
\operatorname{Prob}\left(\operatorname{Kir}\left(K_{n}\right)=d\right)=\frac{1}{q-1} \cdot \frac{\Pi_{2 r}\left(q^{-1}\right)}{\Pi_{r}\left(q^{-2}\right)}\left(1+s q^{-r}\right),
$$


where $r=\lceil(n-1) / 2\rceil$ are as defined in (14) and $s$ is as follows:

$$
s= \begin{cases}0 & \text { if } p \text { or } n \text { is even }, \\ 1 & \text { if }(-1)^{r} d \text { is a quadratic residue in } \mathbb{F}_{q} \\ -1 & \text { otherwise. }\end{cases}
$$

where $\Pi_{k}(x)=\prod_{i=1}^{k}\left(1-x^{i}\right)$,

We note that the Legendre symbol satisfies $\left(\frac{(-1)^{r}}{p}\right)=(-1)^{r(p-1)}$ for $p$ prime, so (16) is exactly (13) when $q$ is a prime $p$.

We consider the distribution of $\operatorname{Kir}\left(K_{n}\right)$, denoted by $D_{n}$. The probability $\operatorname{Prob}\left(\operatorname{Kir}\left(K_{n}\right)=d\right)$ is denoted by $D_{n}(d)$. Let ${ }_{j} D_{n}$ denote the distribution $D^{\prime}$ such that $D^{\prime}(d)=D_{n}\left(j^{-1} d\right)$. Let zero be the always-zero distribution.

Lemma 4.1 For $n \geq 4$, we have the following recurrence:

$$
\begin{aligned}
D_{n}= & \sum_{j \neq 0} \operatorname{Prob}\left(a_{1,1}=j\right) \cdot{ }_{j} D_{n-1}+q^{1-n} \cdot \text { zero } \\
& +\sum_{t} \sum_{j \neq 0} \operatorname{Prob}\left(a_{1,1}=\ldots=a_{1, t-1}=0, a_{1, t}=j \neq 0\right) \cdot{ }_{-j^{2}} D_{n-2} \\
= & \left(1-q^{-1}\right) \bar{D}_{n-1}+q^{1-n} \cdot \text { zero }+\left(\sum_{i=1}^{n-2} q^{-i}\right) \sum_{j \neq 0} q^{-1} \cdot{ }_{-j^{2}} D_{n-2}
\end{aligned}
$$

Here $\bar{D}$ is the uniform distribution over $\mathbb{F}^{*}$ with $\bar{D}(0)=D(0)$. For $d \neq 0$, $\bar{D}(d)=(1-D(0)) /(q-1)$.

Proof: For the random symmetric $(n-1) \times(n-1)$ matrix

$$
A=\left(\begin{array}{cccc}
a_{1,1} & a_{1,2} & \cdots & a_{1, n-1} \\
a_{2,1} & a_{2,2} & \cdots & a_{2, n-1} \\
\vdots & \vdots & \ddots & \vdots \\
a_{n-1,1} & a_{n-1,2} & \cdots & a_{n-1, n-1}
\end{array}\right)
$$

we apply Lemma 3.1 to compute the determinant. We pivot with respect to entries on the first row. The terms in (17) correspond to the following cases:

(i) $a_{1,1} \neq 0$.

(ii) $a_{1, i}=0, \forall i$. 
(iii) All other cases are divided into subcases that $a_{1,1}=\cdots=a_{1, s-1}=0$, but $a_{1, s} \neq 0$. We use the fact that the minor formed by deleting the first and the $s$-th rows and columns is still random symmetric.

Proof of Theorem 15: We will derive equation (15) by applying induction on $r=\left\lceil\frac{n-1}{2}\right\rceil$. For $r=1$, we have $n=2$ or 3 . Clearly, $D_{2}$ is uniformly distributed over $\mathbb{F}_{q}$.

$$
D_{2}(d)=q^{-1}
$$

for all $d$.

When $n=3, D_{3}$ can be checked directly.

$$
D(d)= \begin{cases}q^{-1} & \text { if } d=0 \\ q^{-1}+\left(\frac{-d}{q}\right) q^{-2} & \text { if } d \neq 0 .\end{cases}
$$

Therefore for $r=1$, we know that (15) is true. Now we assume that (15) holds for $r=k$, i.e. $n=2 k$ and $2 k+1$. We now consider $r=k+1$. In the recurrence (17), the first two terms are uniformly distributed over $\mathbb{F}_{q}^{*}$, so is the last term if $n$ or $p$ is even. Hence $D_{2 k+2}$ is uniformly distributed over $\mathbb{F}_{q}^{*}$.

$$
D_{2 k+2}(d)= \begin{cases}\operatorname{Pk}\left(K_{2 k+2}\right)=1-\frac{\Pi_{2 k}\left(q^{-1}\right)}{\Pi_{k}\left(q^{-2}\right)} & \text { if } d=0 \\ \frac{1}{(q-1)} \cdot \frac{\Pi_{2 k}\left(q^{-1}\right)}{\Pi_{k}\left(q^{-2}\right)} & \text { if } d \neq 0\end{cases}
$$

This is consistent with equation (15).

For $n=2 k+3$, we note that $D_{2 k+3}(0)$ is $\operatorname{Pk}\left(K_{2 k+3}\right)$. For $d \neq 0$ and $q$ odd, the first two terms in recurrence (17) are uniformly distributed over $\mathbb{F}_{q}^{*}$. By the induction hypothesis, we compute as follows:

$$
\begin{aligned}
D_{2 k+3}(d)= & \left(1-q^{-1}\right)\left(1-D_{2 k+2}(0)\right) /(q-1) \\
& +\left(q^{-1}+q^{-2}+\cdots+q^{-2 k-1}\right) \sum_{j \neq 0} q^{-1} D_{2 k+1}\left(-j^{-2} d\right) \\
= & q^{-1} \frac{\Pi_{2 k+2}\left(q^{-1}\right)}{\Pi_{k+1}\left(q^{-2}\right)} \\
& +q^{-2}\left(1-q^{-2 k-1}\right) \sum_{j \neq 0} \frac{\Pi_{2 k}\left(q^{-1}\right)}{(q-1) \Pi_{k}\left(q^{-2}\right)}\left(1+\left(\frac{(-1)^{k+1} j^{-2} d}{q}\right) q^{-k}\right) \\
= & \frac{1}{q-1} \cdot \frac{\Pi_{2 k+2}\left(q^{-1}\right)}{\Pi_{k+1}\left(q^{-2}\right)}\left(1+\left(\frac{(-1)^{k+1} d}{q}\right) q^{-k-1}\right)
\end{aligned}
$$


Therefore (15) is true for $r=k+1$, i.e. $n=2 k+2$ or $2 k+3$. By induction, (15) holds for all $n$ and Theorem 4.1 is proved.

\section{$5 \quad$ Problems and remarks}

There are many questions remained unsolved concerning the distribution of the probabilistic Kirchhoff polynomials. For example, for a tree $T_{n}$ on $n$ vertices, we have

$$
\operatorname{Prob}\left(\operatorname{Kir}\left(T_{n}\right)=d\right)= \begin{cases}1-\left(1-q^{-1}\right)^{n-1} & \text { if } d=0 \\ q^{-1}\left(1-q^{-1}\right)^{n-2} & \text { otherwise }\end{cases}
$$

Theorem 4.1 implies that for $n$ even over a finite field $\mathcal{F}$

$$
\operatorname{Prob}\left(\operatorname{Kir}\left(K_{n}\right)=d\right)=\operatorname{Prob}\left(\operatorname{Kir}\left(K_{n}\right)=x d\right)
$$

for all $x$ in $\mathcal{F}-\{0\}$. For $n$ odd,

$$
\operatorname{Prob}\left(\operatorname{Kir}\left(K_{n}\right)=d\right)=\operatorname{Prob}\left(\operatorname{Kir}\left(K_{n}\right)=x^{2} d\right)
$$

It would be of interest to determine for which graphs (19) or (20) hold. In general, we can ask the following question:

Problem 1: For a graph $G$, what is the least integer $k$ such that

$$
\operatorname{Prob}(\operatorname{Kir}(G)=d)=\operatorname{Prob}\left(\operatorname{Kir}(G)=x^{k} d\right)
$$

for any $d$ and any non-zero $x$ in a finite field $\mathcal{F}$ ?

We say that a graph $G$ has index $k$ in $\mathcal{F}$ if $k$ is the answer to the above question. So, $\operatorname{index}\left(K_{2 n}\right)=\operatorname{index}\left(T_{n}\right)=1$ and $\operatorname{index}\left(K_{2 n+1}\right)=2$.

Here we describe a fact which is quite useful for determining the index of some graphs: The Kirchhoff polynomial $\mathrm{Pk}(G)$ is homogeneous of degree $n-1$ if $G$ has $n$ vertices. When the random variables assume values in a field $\mathcal{F}$, there is an one-to-one correspondence between the assignments giving $\operatorname{Kir}(G)=d$ and the assignments giving $\operatorname{Kir}(G)=x^{n-1} d$. The mapping can be described as follows:

$$
\left(w_{1}, w_{2}, \ldots, w_{e}\right) \longleftrightarrow\left(x w_{1}, x w_{2}, \cdots, x w_{e}\right)
$$

This observation implies that

$$
\operatorname{Prob}(\operatorname{Kir}(G)=d)=\operatorname{Prob}\left(\operatorname{Kir}(G)=x^{n-1} d\right)
$$


for any graph $G$ on $n$ vertices and any $x \neq 0$. Therefore, the index of $G$ must be a factor of $n-1$. It would be interesting to know when the index is exactly $n-1$. Although it seems to be quite hard to compute the index of a general graph, for a special family of graphs it could be relatively easier to verify. When the number of vertices is $n=p+1$ for some prime number $p$, and $q \equiv 1(\bmod p)$ is the cardinality of the finite field, the only possible value for the index is 1 or $p=n-1$. If we can provide two distinct probabilities for two nonzero values of $d_{1}$ and $d_{2}$, the index of the graph must then be $n-1$.

Since Dirichlet Theorem asserts that for a fixed $n=p+1$, there are infinitely many primes (hence prime powers) $q$ such that $q \equiv 1(\bmod n-1)$. Therefore we may have infinitely many possible extremal cases.

In particular, we consider cycles $C_{n}$ on $n$ vertices, which have Kirchhoff polynomials

$$
\operatorname{Kir}\left(C_{n}\right)=\sum_{j} \hat{x}_{j}
$$

where $\hat{x}_{i}=\left(\prod_{j} x_{j}\right) / x_{i}$. By computation, we have verified the following small cases:

$n=4, q=7$

$$
\operatorname{Prob}\left(\operatorname{Kir}\left(C_{n}\right)=d\right)= \begin{cases}336 / 7^{4} & \text { when } d=1 \text { or } 6 \\ 294 / 7^{4} & \text { when } d=2 \text { or } 5 \\ 357 / 7^{4} & \text { when } d=3 \text { or } 4 \\ 427 / 7^{4} & \text { when } d=0\end{cases}
$$

$n=4, q=13$

$$
\operatorname{Prob}\left(\operatorname{Kir}\left(C_{n}\right)=d\right)= \begin{cases}2262 / 13^{4} & \text { when } d=2,3,10 \text { or } 11, \\ 2145 / 13^{4} & \text { when } d=4,6,7 \text { or } 9, \\ 2106 / 13^{4} & \text { when } d=5,8,1 \text { or } 12, \\ 2509 / 13^{4} & \text { when } d=0 .\end{cases}
$$


$n=6, q=11$

$$
\operatorname{Prob}\left(\operatorname{Kir}\left(C_{n}\right)=d\right)= \begin{cases}149930 / 11^{6} & \text { when } d=1 \text { or } 10, \\ 151360 / 11^{6} & \text { when } d=2 \text { or } 9, \\ 152185 / 11^{6} & \text { when } d=3 \text { or } 8, \\ 150810 / 11^{6} & \text { when } d=4 \text { or } 7, \\ 150260 / 11^{6} & \text { when } d=5 \text { or } 6, \\ 262471 / 11^{6} & \text { when } d=0 .\end{cases}
$$

Therefore we have proved the following fact:

Lemma 5.1 The index of $C_{4}$ is 3 over the finite field $\mathbb{F}_{7}$ is 3 . or $\mathbb{F}_{13}$. The index of $C_{6}$ over the finite field $\mathbb{F}_{11}$ is 5 .

This leads to the following problem:

Problem 2: Prove or disprove that the index of $C_{p+1}$ is $p$ for a prime $p$ over the finite field $\mathbb{F}_{q}$ with $q \equiv 1(\bmod p)$.

Of course, it would be even better if the index of any cycles can be determined.

Another natural question is whether that $\operatorname{Prob}(\operatorname{Kir}(G)=d$ ) over the finite field $\mathbb{F}_{q}$ is a universal function of $1 / q$ or not. From equation (15), we see that the answer is negative for $d \neq 0, q$ prime, $n$ odd and $G=K_{n}$.

We remark that there are counterexamples for the version of Kontsevich's conjecture over a ring. In [2], the explicit formula for $\operatorname{Prob}(\operatorname{Kir}(G)=$ 0 over $\mathbb{Z}_{q}$ ) for $q=p^{k}$, where $p$ is a prime and $k>1$, is a polynomial in $1 / p$ depending on $k$ but not a universal polynomial in $1 / q$.

So far, we have not found any counterexample to the following strengthened version of Kontsevich's conjecture:

Conjecture : For a graph $G$ and a positive integer $r$, the probability that its reduced combinatorial Laplacian $\tilde{L}$ of $G$ over the finite field $\mathbb{F}_{q}$ has rank $r$, denoted by $\operatorname{Prob}(\operatorname{rank}(\tilde{L}(G))=r)$, is a universal function in $1 / q$.

\section{References}

[1] R. P. Brent and B. D. McKay, On determinants of random matrices over $\mathbb{Z}_{m}$, Discrete Math. 66 (1987), 35-49. 
[2] R. P. Brent and B. D. McKay, Determinants and ranks of random matrices over $\mathbb{Z}_{m}$, Ars Combinatoria 26A (1988), 57-64.

[3] L. Carlitz, Representations by quadratic forms in a finite field, Duke Math. J. 21 (1954), 123-137.

[4] F. Kirchhoff, Über die Auflösung der Gleichungen, auf welche man bei der Untersuchung der linearen Verteilung galvanischer Ströme geführt wird, Ann. Phys. chem. 72 (1847), 497-508.

[5] M. Kontsevich, Gelfand Seminar talk, Rutgers University, December 8, 1997.

[6] J. MacWilliams, Orthogonal matrices over finite fields, Amer. Math. Monthly 76 (1969), 152-164.

[7] R. Stanley, Spanning Trees and a Conjecture of Kontsevich, Annals of Combinatorics 2 (1998), 351-363.

[8] J. R. Stembridge, Counting points on varieties over finite fields related to a conjecture of Kontsevich, Annals of Combinatorics 2 (1998), 365385 .

[9] J. J. Sylvester, On the change of systems of independent variables, Quarterly Journal of Mathematics 1 (1857), 42-56. Collected Mathematical Papers, Cambridge 2 (1908), 65-85.

[10] C. Yang, The probabilistic Kirchhoff polynomials over finite field $\mathbb{F}_{p^{k}}$ and a conjecture of Kontsevich, Ph. D. Thesis, University of Pennsylvania, March 1999. 